J. Clin. Chem. Clin. Biochem.

Vol. 17, 1979, pp. 619-625

\title{
Mikrobestimmung von Lipoproteinlipiden im Serum
}

\author{
Von O. Leiß ${ }^{1}$ ), U. Murawski und H. Egge
}

Institut für Physiologische Chemie der Universität Bonn

(Eingegangen am 8. Dezember 1978/21. Mai 1979)

\begin{abstract}
Zusammenfassung: Durch Kombination einer Mikromethode zur Analyse von Serumlipiden mit bekannten Präzipitationsverfahren zur Isolierung von Lipoproteinen wurde eine Methode entwickelt, die es erlaubt, aus $50 \mu \mathrm{l} \mathrm{Serum}$ eine ausführliche Analyse der Lipidverteilung (Phospholipide, freies Cholesterin, verestertes Cholesterin, Triglyceride und freie Fettsäuren) in den einzelnen Lipoproteinen durchzuführen. Hierzu wurden die VLDL mit Heparin/Mg ${ }^{++}$, die LDL mit Dextransulfat $500 / \mathrm{Mg}^{++}$und die $\mathrm{HDL}$ mit Dextransulfat $/ \mathrm{Mn}^{++}$successive gefällt. Nach Zentrifugation der Präzipitate wurden die Lipide extrahiert und gravimetrisch quantitativ sowie nach dünnschichtchromatographischer Trennung und Veraschung densitometrisch bestimmt (van Gent, C. M. (1968), Z. Anal. Chem. 236, 344-350; Egge, $H$. et al. (1970) Z. Klin. Chem. Klin. Biochem. 8, 488-491). Die aus den Seren von 12 gesunden Probanden erhaltenen Daten wurden mit den Ergebnissen verglichen, die nach entsprechender Lipoproteinisolierung mittels präparativer Ultrazentrifugation erhalten wurden (Havel, $R$. J. et al. (1955) J. Clin. Invest. 34, 1345-1353). Hierbei zeigten die Fraktionen der $\beta$-Lipoproteine $(\mathrm{d}<1,063 \mathrm{~g} / \mathrm{ml})$ und $\operatorname{HDL}(1,063<\mathrm{d}<1,21 \mathrm{~g} / \mathrm{ml})$ gute Übereinstimmung in ihrer relativen Lipidverteilung, während die Fraktionen der VLDL $(\mathrm{d}<1,006 \mathrm{~g} / \mathrm{ml})$ und der VHDL $(\mathrm{d}>1,21 \mathrm{~g} / \mathrm{ml})$ weniger gut mit den entsprechenden Fraktionen nach Fällung übereinstimmten. Bezogen auf die Gesamtlipide des Serums betrug die Wiederfindungsrate $95-105 \%$. Die Variationskoeffizienten für die einzelnen Lipidklassen liegen bei den VLDL-Lipiden bei 15-20\%, bei den LDL- und HDLLipiden bei 5-10\% und bei den VHDL-Lipiden bei 10-15\%. Für das Gesamtlipid, das der Quantifizierung der Einzelkomponenten zugrundegelegt wird, lag der Variationskoeffizient bei den LDL bei $4 \%$ und bei den HDL bei $6 \%$.
\end{abstract}

\section{The microanalysis of serum lipoprotein lipids}

Summary: A method is described which allows the determination of phospholipids, free and esterified cholesterol, triglycerides and free fatty acids in lipoprotein fractions starting from $50 \mu \mathrm{l}$ of serum. Lipoproteins were separated by successive precipitation: VLDL with Heparin $/ \mathrm{Mg}^{++}, \mathrm{LDL}$ with Dextran sulfate $/ \mathrm{Mg}^{++}$and finally $\mathrm{HDL}$ with Dextran sulfate $/ \mathrm{Mn}^{++}$. Lipids extracted from the precipitated lipoproteins were determined gravimetrically and by densitometry after thin layer chromatography and charring (van Gent, C.M. (1968), Z. Anal. Chem. 236, 344-350; Egge, H. et al. (1970) Z. Klin. Chem. Klin. Biochem. 8, 488-491). The results obtained from the serum of 12 adult healthy persons were compared with those from lipoprotein fractions separated by preparative ultracentrifugation (Havel, R. $J$. et al. (1955) J. Clin. Invest. 34, 1345-1353). The distribution of lipids in $\beta$-lipoproteins $(\mathrm{d}<1.063 \mathrm{~g} / \mathrm{ml})$ and $\mathrm{HDL}$ $(1.063<\mathrm{d}<1.21 \mathrm{~g} / \mathrm{ml})$ prepared by both methods showed good agreement. Some differences were observed between VLDL $(\mathrm{d}<1.006 \mathrm{~g} / \mathrm{ml})$ and VHDL $(\mathrm{d}>1.21 \mathrm{~g} / \mathrm{ml})$ prepared either by precipitation or ultracentrifugation. Compared to the total lipid of the sera, recovery rates were $95-105 \%$. Variation coefficients were in the range of $15-20 \%$ for VLDL lipids, 5-10\% for LDL and HDL lipids and 10-15\% for VHDL lipids. Gravimetrically determined total lipids had a variation coefficient of 4 and $6 \%$ for $\mathrm{LDL}$ and $\mathrm{HDL}$ respectively.

\section{Einführung}

Hyperlipoproteinämien gehören zu den häufigsten Stoffwechselkrankheiten und stellen einen Risikofaktor erster Ordnung für Coronarerkrankungen dar (1-4). Die Charakterisierung der Hyperlipoproteinämietypen erfolgt mittels verschiedener Methoden (5-7), wobei die präparative Ultrazentrifugation in Kombination mit Lipidanalysen der getrennten Fraktionen als Referenzmethode dient. Für die klinische Routine ist sie jedoch zu teuer und zu zeitintensiv. Zur phänotypischen Klassifizierung der Hyperlipoproteinämien $(8,9)$ dient die Lipoproteinelektrophorese $(8,10)$. Sie erlaubt jedoch nur eine qualitative bzw. semiquantitative Beurteilung der Lipoproteinverteilung und wird neuerdings für überflüssig gehalten

\footnotetext{
1) Neue Anschrift: Medizinische Klinik der Universität Bonn, Bonn-Venusberg.
} 
$(11,12)$. Neben immunologischen Methoden zur quantitativen Bestimmung von Apolipoprotein B $(13,14)$ und Apolipoprotein A-I und A-II (15) wurden verschiedene Präzipitationsmethoden entwickelt $(16,17)$. Sie beruhen meist auf einer Fällung der Apolipoprotein B enthaltenden Lipoproteine mittels Polyanionen wie Heparin, Dextransulfat u.ä. in Gegenwart zweiwertiger Kationen wie $\mathrm{Ca}^{++}$, $\mathrm{Mg}^{++}$oder $\mathrm{Mn}^{++}$.

\section{Da niedrige $\mathrm{HDL}^{2}$ )-Cholesterin-Konzentrationen mit} einer erhöhten Incidenz für ischämische Coronarerkran= kungen (18-22) und Cerebralerkrankungen (23) einhergehen und umgekehrt erhöhte HDL-Cholesterin-Werte, wie z.B. bei der familiären Hyper- $\alpha$-lipoproteinämie, für ein vermindertes coronares Risiko und eine erhöhte Lebenserwartung zu sprechen scheinen (24), gewinnen besonders Methoden zur Quantifizierung der HDL in jüngster Zeit zunehmend an Bedeutung (25-27).

Diese Methoden erlauben eine quantitative Differenzierung in die ,atherogenen “ $\beta$-Lipoproteine und die ,antiatherogenen " $\alpha$-Lipoproteine. Die weitere Unterscheidung der prä- $\beta$-Lipoproteine (VLDL) und der $\beta$-Lipoproteine (LDL) ist vor allem für die Differenzierung der häufigsten Hyperlipoproteinämien vom Typ II b und IV von großer klinischer Bedeutung.

Hierfür wurden in den letzten Jahren von Wilson \& Spiger (28) und Ononogbu \& Lewis (29) Fällungsmethoden in Parallelansätzen vorgeschlagen, die, kombiniert mit automatisierten Triglycerid- und Cholesterinbestimmungen, durch geeignete Differenzbildung indirekt Aussagen über Cholesterin- und Triglyceridgehalt der VLDL, LDL und HDL erlauben.

In dieser Arbeit werden Fällungsmethoden beschrieben, die ausgehend von $50 \mu$ l Serum eine schnelle Quantifizierung der Lipoproteine und deren Lipidkomponenten erlauben.

\section{Methodik}

Probanden

Von 4 weiblichen und 8 männlichen gesunden normalgewichtigen Probanden (Alter 24-42 Jahre) wurden nach 14-stündigem Fasten jeweils $20 \mathrm{ml}$ Blut aus der Cubitalvene entnommen und zur $\mathrm{Ge}$ rinnung 2 Stunden bei Raumtemperatur stehengelassen. Von dem durch $15 \mathrm{~min}$. Zentrifugation bei $3000 \mathrm{U} / \mathrm{min}$ abgetrennten Serum wurden $50 \mu \mathrm{l}$-Aliquots in vorbereitete Eppendorf-Gefäße

\footnotetext{
2) Abkürzungen:

VLDL $=$ very low density lipoproteins $(\mathrm{d}=<1,006 \mathrm{~g} / \mathrm{ml})$

$\mathrm{LDL}=$ low density lipoproteins $\quad(\mathrm{d}=1,006-1,063 \mathrm{~g} / \mathrm{ml})$

IDL $=$ intermediate density lipoproteins

$(\mathrm{d}=1,006-1,019 \mathrm{~g} / \mathrm{ml})$

$\mathrm{HDL}=$ high density lipoproteins $\quad(\mathrm{d}=1,063-1,21 \mathrm{~g} / \mathrm{ml})$

VHDL $=$ very high density lipoproteins $(\mathrm{d}=>1,21 \mathrm{~g} / \mathrm{ml})$

$\mathrm{CHE}=$ Cholesterinester

TG = Triglyceride

$\mathrm{CH}=$ freies Cholesterin

FFA = freie Fettsäuren

$\mathrm{PL}=$ Phospholipide

$\mathrm{GCH}=$ Gesamtcholesterin

$\mathrm{GL} \quad=$ Gesamtlipid
}

transferiert und bis zur weiteren Analyse bei $-20^{\circ} \mathrm{C}$ tiefgefroren Jeweils 5 ml-Aliquots wurden direkt zur Lipoproteinisolierung mittels präparativer Ultrazentrifugation verwandt.

Darstellung der Lipoproteine, quantitative Analyse der Lipide

Reagenzien

Dextransulfat 500 (Serva, Heidelberg)

Liquemin ${ }^{(R)} 10$ 000-Ampullen (Hoffmann La-Roche), alle übrigen Reagenzien (p.a.). wurden von der Fa. Merck, Darmstadt bezogen.

\section{Geräte}

Beckman L 2-Ultrazentrifuge

Pyknometer

Christ-Zentrifuge, Photometer, Abdampfapparatur

Eppendorf-Mikroliter-System 3000

$10 \mu \mathrm{l}$-Mikroliterspritze (Fa. Hamilton)

$50 \mu$ l-Mikroliterspritze (Fa. Hamilton, ESGE bzw. Terumo)

Metallblock und Eisbad

Cahn-Gram Elektrowaage (Wägebereich 0,1-1000 $\mu$ g, Präzision:

$\pm 0,1 \mu \mathrm{g})$

Heizplatten $140^{\circ} \mathrm{C}$

Aluminiumschälchen $\emptyset 14 \mathrm{~mm}$

Die verwendeten Eppendorf-Gefäße (Volumen 1,5 ml) und Pipettenspitzen wurden zur Entfernung von Weichmachern 8 Stunden im Soxhlet mit Chloroform/Methanol (Volumina, 1 l + 1 l) extrahiert und anschließend 3 Stunden bei $90^{\circ} \mathrm{C}$ getrocknet (31).

\section{Ultrazentrifugation}

Die Ultrazentrifugation wurde nach der Methode von Havel et al. (32) durchgeführt. Zur VLDL-Trennung wurden 3-5 ml frisches Serum mit $0,15 \mathrm{~mol} / 1 \mathrm{NaCl}(\mathrm{d}=1,006 \mathrm{~g} / \mathrm{ml})$ überschichtet und in einem Ti 50-Rotor 20 Stunden bei $15^{\circ} \mathrm{C}$ und $105000 \mathrm{~g}$ in einer Beckman-Spinco L2-Ultrazentrifuge zentrifugiert. Zur $\beta$ Lipoproteintrennung (VLDL+LDL) wurden $5 \mathrm{ml}$ Serum mit $\mathrm{KBr}$ auf die Dichte $\mathrm{d}=1,063 \mathrm{~g} / \mathrm{ml}$ eingestellt und 20 Stunden bei $15^{\circ} \mathrm{C}$ und $105000 \mathrm{~g}$ zentrifugiert. Bei einem Teil der Proben wurde anschließend zusätzlich ein Aliquot des Unterstandes $\mathrm{d}=>1,063 \mathrm{~g} / \mathrm{ml}$ auf $\mathrm{d}=1,21 \mathrm{~g} / \mathrm{ml}$ eingestellt und zur Isolierung der HDL $24 \mathrm{~h}$ bei $105000 \mathrm{~g}$ zentrifugiert. Die erhaltenen Über(HDL) und Unterstände (UZ-Rest; $\mathrm{d}=>1,21 \mathrm{~g} / \mathrm{ml}$ ) wurden ebenso wie alle anderen Lipoproteinfraktionen bis zur weiteren Analyse (innerhalb $24 \mathrm{~h}$ ) im Kühlschrank bei $4^{\circ} \mathrm{C}$ aufbewahrt. Die Dichte der verwendeten Salzlösungen wurde pyknometrisch kontrolliert.

\section{Chemische Analysen}

Zur quantitativen Cholesterin-Bestimmung wurden die einzelnen Lipoproteinfraktionen mit Chloroform/Methanol nach Folch et al. (33) extrahiert. Der Lipidextrakt wurde jeweils über eine Kieselgèlsäulè (HR 60) gereinigt. Hierzu wurden $5 \mathrm{~g}$ Kieselgel HR 60 in $25 \mathrm{ml}$ Chloroform aufgeschlämmt und nach vollständiger Homogenisierung davon $1 \mathrm{ml}$ in ein kapillar ausgezogenes Glasrohr ( $\emptyset 7 \mathrm{~mm}$, Höhe $15 \mathrm{~cm}$ ) transferiert. Nach Waschen der Säule mit $3 \mathrm{ml}$ Chloroform wurde die Lipidprobe aufgegeben und mit $20 \mathrm{ml}$ Chloroform eluiert. Das Eluat wurde unter Stickstoff zur Trockne gebracht und das Cholesterin nach der Methode von Zlatkis et al. (34) bestimmt. Die Wiederffindungsrate bei in gleicher Weise über Säule gereinigten Standards betrug $95-100 \%$.

Die relative Lipidverteilung der einzelnen Lipoproteinfraktionen wurde densitometrisch $(30,31)$ ermittelt.

\section{Fällung der Lipoproteine}

Die in vorextrahierten (31) Eppendorf-Gefäßen tiefgefrorenen Serumproben $(50 \mu \mathrm{l})$ wurden zum Auftauen $15 \mathrm{~min}$ bei Raumtemperatur belassen und anschließend im Metallblock im Eisbad inkubiert.

Zur VLDL- und LDL-Fällung wurde $1 \mathrm{ml}$ Liquemin(R) 10000 mit $1 \mathrm{ml}$ bidest. Wasser und $2 \mathrm{ml}$ einer $2 \mathrm{~mol} / \mathrm{l} \mathrm{MnCl}_{2}$ Lösung versetzt. Hiervon wurden $5 \mu \mathrm{l} \mathrm{zu} 50 \mu \mathrm{l}$ Serum gegeben (Endkonz.: $250000 \mathrm{USP}-\mathrm{E} / 1 \mathrm{Heparin}$ und $0,045 \mathrm{~mol} / \mathrm{l} \mathrm{MnCl}_{2}$ ) und $20 \mathrm{~s}$ geschüttelt. Nach 30 min Inkubation bei $0^{\circ} \mathrm{C}$ wurde $2 \mathrm{~min}$ bei $15000 \mathrm{~g}$ zentrifugiert. Der klare Überstand wurde mit einer $50 \mu \mathrm{l}$-Mikroliterspritze aspiriert und in ein zweites Reaktionsge- 
fäß transferiert. Aus Sediment und Uberstand wurden die Lipide mit jeweils $500 \mu \mathrm{l}$ Chloroform/Methanol (Volumina, $80 \mathrm{ml} \mathrm{+}$ $50 \mathrm{ml}$ ) nach Folch et al. (32) extrahiert. Zur successiven Lipoproteinfällung (Schema 1) wurden die Stammlösungen Heparin (5000 units/ml), $\mathrm{MgCl}_{2}$ (2 mol/1), Dextransulfat $500(20 \mathrm{~g} / \mathrm{l})$, Dextransulfat $500(150 \mathrm{~g} / \mathrm{l})$ und $\mathrm{MnCl}_{2}(5 \mathrm{~mol} / \mathrm{l})$ hergestellt und im Kühlschrank aufbewahrt.

Zur VLDL-Fällung wurde die Heparinlösung (5000 units/ml) mischt. Genau $5 \mu$ dieser Lösung wurden bei eingetauchter Kanülenspitze langsam zu $50 \mu l$ Serum gegeben (Endkonz. Heparin 250000 USP-E/1, $\mathrm{Mg}^{++} 0,09 \mathrm{~mol} / \mathrm{l}$ ). Nach Durchmischen wurde $30 \mathrm{~min}$ bei $0^{\circ} \mathrm{C}$ inkubiert und zentrifugiert. Der Überstand wurde vorsichtig von der Oberfläche her und mit Kanülenöffnung an der dem Sediment abgewandten Wand des Reaktionsgefäßes möglichst durch zweimaliges Aspirieren von jeweils $25-30 \mu l$ in ein zweites vorgekühltes Reaktionsgefäß überführt. Bei Seren nüchterner gesunder Personen verblieb am Boden des Reaktionsgefäßes ein diffus-trüber Niederschlag.

Bei Seren mit erhöhter Triglyceridkonzentration trat schon bei Zugabe von Heparin-Magnesium eine starke Trübung auf. Der entstandene Heparin-VLDL-Komplex flotierte 2.T. bei Zentrifugation über einem trüben Unterstand. Es empfiehlt sich daher, starke hyperlipämische Seren vor Analyse mit bidest. Wasser zu verdünnen, um eine vollständige Fällung und einen klaren Überstand zu erhalten.

Zur LDL-Fällung wurden die Lösungen Dextransulfat 500 $(20 \mathrm{~g} / \mathrm{l})$ und $\mathrm{MgCl}_{2}(2 \mathrm{~mol} / \mathrm{l})$ zu gleichen Teilen vermischt. $5 \mu \mathrm{l}$ dieser frischen Lösung wurden zu dem klaren Überstand nach VLDL-Fällung gegeben (Endkonz. Dextransulfat $500 \sim 0,9 \mathrm{~g} / \mathrm{l}$, $\left.\mathrm{MgCl}_{2} \sim 0,09 \mathrm{~mol} / \mathrm{l}\right)$. Nach Durchmischen, 30 min Inkubation bei $0^{\circ} \mathrm{C}$ und Zentrifugation $(15000 \mathrm{~g}, 2 \mathrm{~min}$ ) wurde über einem scharfen Sediment ein klarer Überstand erhalten. Dieser wurde wie oben in ein drittes Reaktionsgefäß transferiert.

Zur Fällung der HDL wurden Dextransulfat $500(150 \mathrm{~g} / \mathrm{l})$ und mit dem gleichen Volumen einer $2 \mathrm{~mol} / 1 \mathrm{MgCl}_{2}$ Lösung ver$\mathrm{MnCl}_{2}(5 \mathrm{~mol} / \mathrm{l}) \mathrm{zu}$ gleichen Teilen vermischt. $5 \mu \mathrm{l}$ dieser fri-

schen Lösung wurden zum Überstand nach LDL-Fällung zugegeben (Endkonz. Dextransulfat $500 \sim 6,8 \mathrm{~g} / \mathrm{l}, \mathrm{MnCl}_{2} \sim 0,22$ mol/l) (17). Nach Durchmischen, Inkubation $30 \mathrm{~min}$ bei $0^{\circ} \mathrm{C}$ und Zentrifugation wurde der Uberstand in ein viertes Reaktionsgefäß transferiert.

\section{Lipidextraktion und gravimetrische Gesamtlipidbestim- mung}

$\mathrm{Zu}$ den gefällten Lipoproteinen wurden jeweils $500 \mu \mathrm{l}$ Chloroform/Methanol (Volumina, $80 \mathrm{ml}+50 \mathrm{ml}$ ) zugegeben, zum Überstand nach HDL-Fällung (VHDL) zusätzlich noch $50 \mu$ l Methanol. Nach 1 min Rütteln bei Raumtemperatur und Zentrifugation ( $2 \mathrm{~min}$ bei $15000 \mathrm{~g}$ ) wurde der Uberstand mit einer kapillar ausgezogenen Pasteur-Pipette in ein weiteres Reaktionsgefäß transferiert. Die Extraktion wurde mit $500 \mu \mathrm{l}$ Chloroform/ Methanol wiederholt. Die vereinigten Überstände wurden mit $200 \mu$ l bidest. Wasser versetzt, 30 s gerüttelt und anschließend 30 s zentrifugiert. Nach Waschen des Extraktes mit $200 \mu \mathrm{l} \mathrm{bi-}$ dest. Wasser wurde die obere wäßrige Phase abgehoben und verworfen. Ein eventuell auftretender Niederschlag an der Grenzschicht muß sorgfältig entfernt werden. Nach Eindampfen unter $\mathrm{N}_{2}$ wurden die Lipide in exakt $100 \mu l$ (Mikroliterspritze) Chloroform/Methanol (Volumina, $20 \mathrm{ml}+10 \mathrm{ml}$ ) gelöst. Die gravimetrische Bestimmung des Gesamtlipids erfolgte aus jeweils $25 \mu \mathrm{l} \mathrm{bzw}$. $50 \mu \mathrm{l}$ bei den LDL-Lipiden. Gesamtlipidbestimmung, Dünnschichtchromatographie und Densitometrie wurden im Detail bereits (31) beschrieben.

\section{Auswertung}

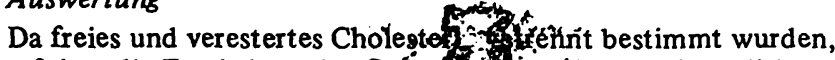
erfolgte die Ermittlung des Gesion of lesterins durch Addition des Cholesteringehaltes in den, Clig terinestern (=Cholesterinester $\times 0,56$ ) und des freien Cholest

Aus dem jeweiligen Gesamtlinito sullen Lipoproteine

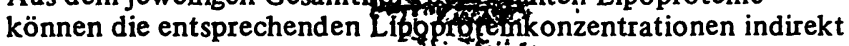
approximativ ermittelt werden. Hiezż wurden bei den VLDL

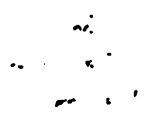

Schema 1. Lipoprotein-Fraktionierung

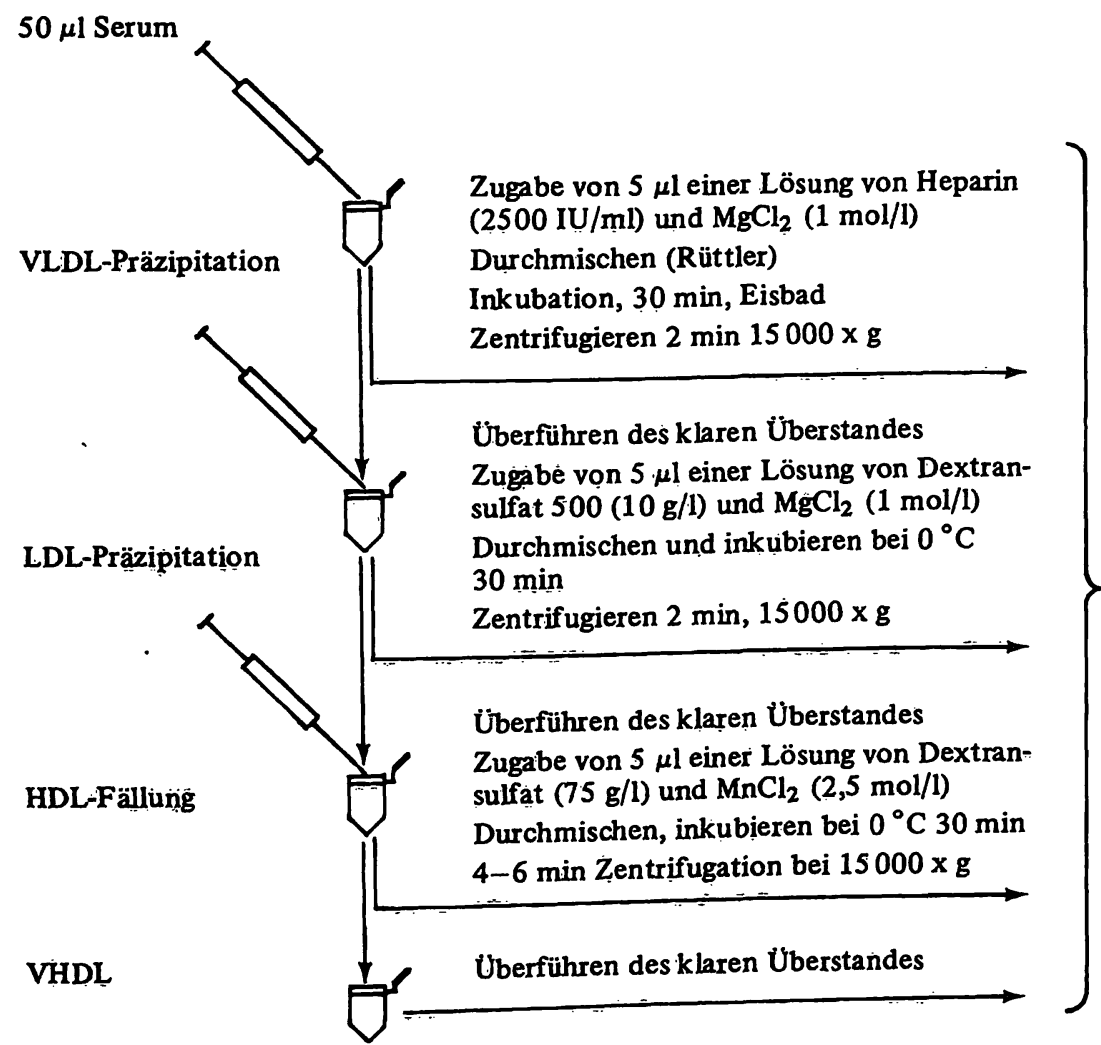

Quantifizierung der Lipide
1. Zweimalige Extriktion der Lipide nach Folch (33) …
2. Waschen des Extraktes
3. Trocknen des Extraktes unter $\mathrm{N}_{2}$
4. Auflösen in $100 \mu / \mathrm{CHCl}_{3}: \mathrm{MeOH}=2: 1$
5. Gravimetrische Bestimmung des Gesamt- lipids aus einem Aliquot
6. Trennung der Lipîte durch Dünnschicht- chromatographie
7. Densitometrische Bestimmung der Lipid- verteilung nach Veraschen (31) 
ein durchschnittlicher Proteinanteil von 10\%, bei den LDL ein Proteinanteil von $25 \%$, bei den HDL ein Proteinanteil von $50 \%$ $(37,38)$ und bei den VHDL von $98 \%$ zugrundegelegt $(38)$. Alle Analysen wurden doppelt durchgefürt. Die Berechnung von Standardabweichung und Variationskoeffizient erfolgte nach bekannten statistischen Methoden.

\section{Ergebnisse}

Tabelle 1 zeigt die prozentuale Lipidzusammensetzung verschiedener Lipoproteinfraktionen nach Ultrazentrifugation und nach Fällung. Die mit der Fällungsmethode erhaltene Relativverteilung der Gesamt- $\beta$-(VLDL+LDL) und $\alpha$-Lipoproteine stimmt gut mit derjenigen nach Ultrazentrifugation überein, während die prozentuale Lipidzusammensetzung der VLDL nach Ultrazentrifugation bei $\mathrm{d}=1,006 \mathrm{~g} / \mathrm{ml}$ und nach Fällung mit Heparin- $\mathrm{MgCl}_{2}$ stärker differiert. Die gefällten VLDL enthalten mehr Cholesterinester und weniger Triglyceride + freie Fettsäuren als diejenigen nach Ultrazentrifugation.

Die prozentuale Lipidzusammensetzung der HDL nach Isolierung in der Ultrazentrifuge $(\mathrm{d}=1,063-1,21 \mathrm{~g} / \mathrm{ml})$ und nach Fällung mit Dextransulfat- $\mathrm{MnCl}_{2}$ stimmt ebenfalls - bis auf die Phospholipide - relativ gut überein, die VLDL dagegen differieren stärker.
Wie aus der Tabelle ferner ersichtlich, wurden im Úberstand nach HDL-Fällung (VHDL) sowohl relativ (in \% der wiedergefundenen Gesamtphospholipide) als auch absolut (in mg/dl) geringfügig mehr Phospholipide gefunden als von anderen Autoren mittels Elektrophorese, Ultrazentrifugation und Polyanion-Fällung. Typische Densitogramme für die einzelnen Lipoproteinfraktionen sind in Abbildung 1 wiedergegeben.

Aus Tabelle 2 sind die Wiederfindungsraten nach schrittweiser Lipoproteinfällung ersichtlich. Hierzu wurden die jeweiligen mg-Werte der verschiedenen Lipidkomponenten nach Fällung addiert und mit den entsprechenden Werten der in einem Parallelansatz durchgeführten Lipidbestimmung des Gesamtserums (31) verglichen. Die Wiederfindungsraten liegen in Durchschnitt bei 95-105\%. Die Streuung beträgt für die einzelnen Lipidkomponenten (außer freie Fettsäuren) jeweils $\pm 10 \%$ und liegt damit in der Größenordnung der Ultrazentrifugen-Methode (7).

Die Variationskoeffizienten der dreischrittigen Fällungsmethode (Tab. 2) liegen bei den VLDL-Lipiden zwischen $15-20 \%$, bei den LDL- und HDL-Lipiden zwischen 5-10\% und bei den VHDL-Lipiden zwischen 10-15\%. Für das Gesamtlipid, das der Quantifizierung der Ein-

Tab. 1. Lipidzusammensetzung verschiedener Lipoprotein-Fraktionen nach İsolierung in der Ultrazentrifuge und nach Polyanionfällung (Mittelwerte nach Doppelbestimmungen von 12 verschiedenen Seren 4 \$, 8 o).

* Die Wiederfindung nach Ultrazentrifugation und anschließender Entsalzung und Reinigung der Lipidextrakte über eine Kieselgelsäule (HR 60) betrug für Gesamtcholesterin aus HDL + (LDL + VLDL) 87,4\%. Die angegebenen Werte sind für $100 \%$ Wiederfindung korrigiert.

\begin{tabular}{|c|c|c|c|c|}
\hline & Ultrazentrifuge & $\begin{array}{l}\text { Fällung } \\
\text { (\% vom Gesamtlipid) }\end{array}$ & Ultrazentrifuge & Fällung \\
\hline & $\mathrm{d}<1,006 \mathrm{~g} / \mathrm{ml}$ & VLDL & \multicolumn{2}{|c|}{$1,063<\mathrm{d}<1,21 \mathrm{~g} / \mathrm{ml} \mathrm{HDL}$} \\
\hline $\begin{array}{l}\text { Cholesterinester } \\
\text { Triglyceride }\end{array}$ & $18,6 \pm 2,6$ & $29,6 \pm 3,8$ & $\begin{array}{r}34,6 \pm 5,8 \\
9,4 \pm 1,8\end{array}$ & $\begin{aligned} 32,8 & \pm 1,9 \\
8,2 & \pm 2,1\end{aligned}$ \\
\hline Triglyceride + freie Fettsäuren & $43,6 \pm 7,8$ & $31,7 \pm 5,1$ & & \\
\hline $\begin{array}{l}\text { Cholesterin } \\
\text { Freie Fettsäuren }\end{array}$ & $11,2 \pm 1,9$ & $11,7 \pm 0,8$ & $\begin{aligned} 10,3 & \pm 1,6 \\
5,4 \pm & 1,6\end{aligned}$ & $\begin{array}{l}9,9 \pm 1,1 \\
4,4 \pm 1,6\end{array}$ \\
\hline Phospholipide & $26,3 \pm 6,0$ & $27,1 \pm 4,6$ & $40,2 \pm 5,2$ & $44,7 \pm 3,3$ \\
\hline \multirow[t]{2}{*}{ Gesamtcholesterin (mg/dl) } & $17,8 \pm 7,9$ & $13,0 \pm 3,4$ & & \\
\hline & $\left.\mathrm{d}<1,063 \mathrm{~g} / \mathrm{ml}^{*}\right)$ & VLDL + LDL & $\mathrm{d}>1,21 \mathrm{~g} / \mathrm{ml}$ & HDL Überstand (VHDL) \\
\hline $\begin{array}{l}\text { Cholesterinester } \\
\text { Triglyceride } \\
\text { Cholesterin } \\
\text { Freie Fettsäuren } \\
\text { Phospholipide }\end{array}$ & $\begin{array}{r}38,0 \pm 2,7 \\
13,7 \pm 2,0 \\
13,2 \pm 0,9 \\
4,2 \pm 1,4 \\
30,9 \pm 2,3\end{array}$ & $\begin{array}{r}36,7 \pm 2,2 \\
14,8 \pm 1,9 \\
13,2 \pm 0,7 \\
4,4 \pm 1,5 \\
30,9 \pm 2,5\end{array}$ & $\begin{array}{r}20,0 \pm 3,7 \\
10,8 \pm 3,7 \\
7,6 \pm 1,8 \\
22,1 \pm 4,3 \\
39,6 \pm 6,1\end{array}$ & $\begin{aligned} 27,8 \pm & 3,1 \\
6,5 \pm & \pm 1,1 \\
6,9 \pm & 0,4 \\
15,7 \pm & 3,9 \\
43,1 \pm & 2,7\end{aligned}$ \\
\hline \multirow[t]{2}{*}{ Gesamtcholesterin (mg/dl) } & $112,3 \pm 17,3$ & $113,7 \pm 10,8$ & & \\
\hline & $\mathrm{d}>1,063 \mathrm{~g} / \mathrm{ml}^{*} ?$ & $\mathrm{HDL}+\mathrm{VHDL}$ & \multicolumn{2}{|c|}{ Phospholipide der VHDL (HDL-Überstand) } \\
\hline $\begin{array}{l}\text { Cholesterinester } \\
\text { Trigly ceride } \\
\text { Cholesterin } \\
\text { Freie Fettsäuren } \\
\text { Phospholipide }\end{array}$ & $\begin{aligned} & 31,7 \pm 2,8 \\
& 6,7 \pm 2,2 \\
& 9,0 \pm 1,0 \\
& 9,3 \pm 2,0 \\
& 43,3 \pm 2,9\end{aligned}$ & $\begin{array}{r}30,3 \pm 1,8 \\
8,2 \pm 2,1 \\
8,9 \pm 2,0 \\
11,0 \pm 2,9 \\
41,7 \pm 3,8\end{array}$ & $\begin{array}{l}\text { (\%der Phospholipide des } \\
10-1.5(31,38) \\
(\mathrm{mg} / 100 \mathrm{ml}) \\
30(31,38)\end{array}$ & $\begin{array}{l}\text { s Gesamtserums) } \\
16,8 \pm 4,1\end{array}$ \\
\hline Gesamtcholesterin (mg/dl) & $59,7 \pm 11,0$ & $59,7 \pm 9,7$ & . & \\
\hline
\end{tabular}



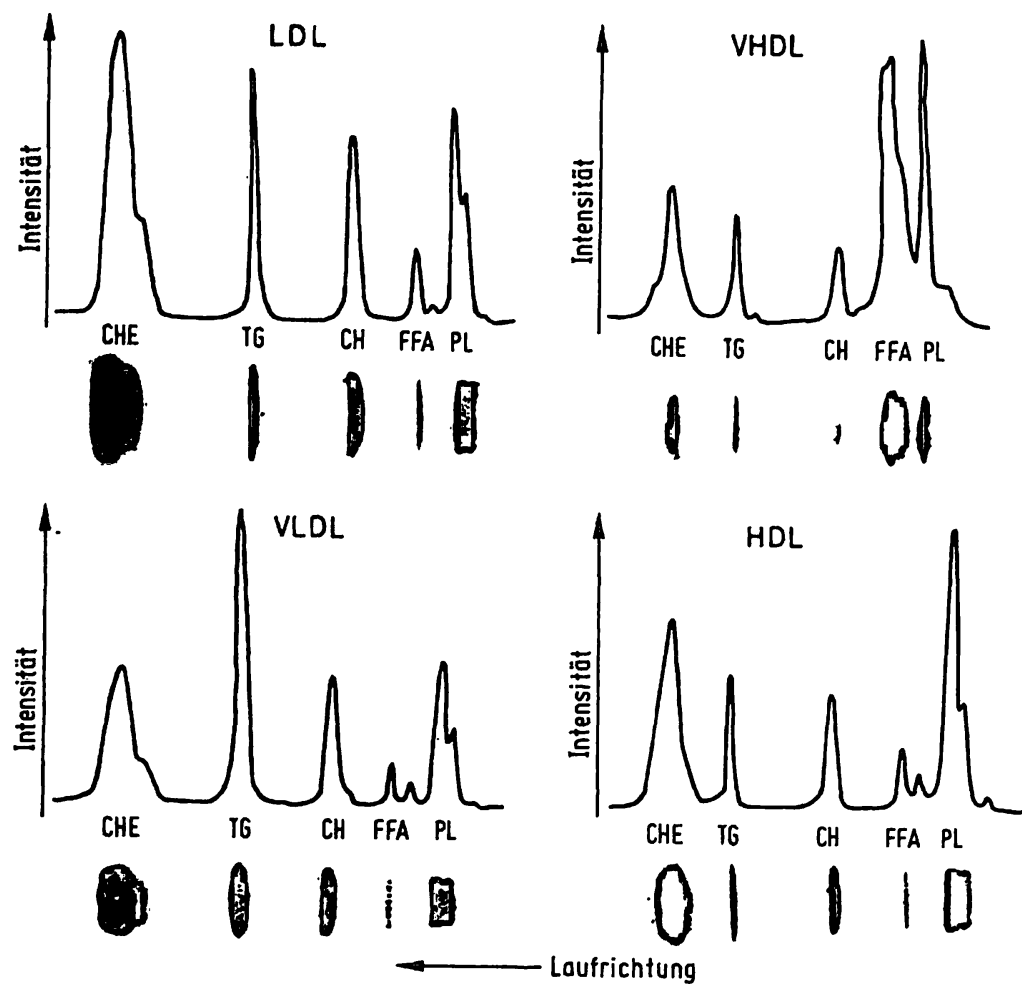

Abb. 1. Dünnschichtchromatographische Trennung der Lipide aus den gefällten Lipoproteinen mit Densitogrammen nach Veraschen.

Tab. 2. Variationskoeffizienten für die einzelnen Lipidkomponenten (in $\mathrm{mg} / \mathrm{dl}$ ) der verschiedenen Lipoproteinfraktionen ( $\mathrm{n}=10$ ) und Wiederfindung der einzelnen Lipidkomponenten nach successiver Fällung in \% des Gesamtserums (Doppelbestimmung von 12 Seren).

* Das Gesamtlipid betrug für die VLDL $=36,9 \mathrm{mg} / \mathrm{dl}$, für die $\mathrm{LDL}=301 \mathrm{mg} / \mathrm{dl}$, für die HDL $=203 \mathrm{mg} / \mathrm{dl}$ und für die VHDL $=99,4 \mathrm{mg} / \mathrm{dl}$ (Serum einer weiblichen Versuchsperson). Die prozentuale Verteilung gleicht den in Tab. 1 angegebenen Werten.

\begin{tabular}{|c|c|c|c|c|c|c|}
\hline \multicolumn{5}{|c|}{ Successive Fällungsmethode*) Variationskoeffizienten } & \multicolumn{2}{|c|}{ Wiederfindungsraten (\%) } \\
\hline & VLDL & LDL & HDL & VHDL & Summe Fällung & Bereich \\
\hline $\begin{array}{l}\text { Cholesterinester } \\
\text { Triglyceride } \\
\text { Cholesterin } \\
\text { Freie Fettsäuren } \\
\text { Phospholipide } \\
\text { Gesamtlipide } \\
\text { Gesamtcholesterin }\end{array}$ & $\begin{array}{l}21,5 \\
15,3 \\
17,0 \\
24,4 \\
17,0 \\
14,3 \\
17,9\end{array}$ & $\begin{array}{r}6,2 \\
8,4 \\
8,3 \\
18,1 \\
7,3 \\
3,8 \\
5,6\end{array}$ & $\begin{array}{r}9,3 \\
10,9 \\
5,4 \\
13,7 \\
6,8 \\
5,9 \\
6,9\end{array}$ & $\begin{array}{r}13,4 \\
16,3 \\
12,7 \\
8,5 \\
14,4 \\
9,9 \\
11,5\end{array}$ & $\begin{array}{r}102,6 \pm 9,1 \\
101,6 \pm 9,1 \\
100,4 \pm 10,8 \\
145,3 \pm 30,9 \\
91,9 \pm 9,1 \\
99,7 \pm 4,3 \\
101,3 \pm 6,5\end{array}$ & $\begin{array}{l}84,8-117,3 \\
90,5-117,7 \\
82,0-116,2 \\
98,7-208,2 \\
80,3-110,4 \\
95,2-108,9 \\
90,3-113,8\end{array}$ \\
\hline
\end{tabular}

zelkomponenten zugrunde gelegt wird, liegt der Variationskoeffizient bei den LDLL bei $4 \%$ und bei den Hị bei $6 \%$.

Tabelle 3 zeigt ein Serum mit leicht erhöhtem Gesamtcholesterin, bei dem nach Lipoproteinanalyse mittels successiver Fällung auf eine Hyperlipoproteinämie Typ II A mit erhöhtem LDL-Cholesterin geschlossen werden konnte.

Wie aus Tabelle 1 ersichtlich, differieren die VLDL- und VHDL-Lipoproteine nach Fällung und nach Ultrazentrifugation in ihrer relativen Lipidzusammensetzung. Die Differenzen in der VLDL-Lipidzusammensetzung sind wahrscheinlich methodisch bedingt. Während die Lipoproteinpartikel in der Ultrazentrifuge entsprechend ihrer Dichte getrennt werden, spielen für die Fällung Oberflächeneigenschaften eine wichtige Rolle. Wie von anderen Arbeitsgruppen gezeigt wurde, sind neben den Phosphilipiden $(39,40)$ positiv geladene Bereiche auf der Oberfläche der Apolipoproteine B und E für die Bindung mit Polyanionen wie Heparin oder Dextransulfat verantwortlich $(41,42)$. Beim Abbau der VLDL reichern sich nach Abspaltung von Apolipoprotein C die Apolipoproteine $B$ und $E$ an der Oberfläche relativ zu den Phospholipiden an (37). Hierbei wird auch die elektrophoretische Mobilität der Partikel beeinflußt, so daß neben den 
Tab. 3. Serum mit erhöhtem Gesamtcholesterin. Die zweischrittige Fällung weist auf eine Hyperlipoproteinämie Typ IIa hin (LDLCholesterin $=209 \mathrm{mg} / \mathrm{dl}$ ). (Doppelbestimmung).

\begin{tabular}{|c|c|c|c|c|c|c|c|c|c|c|}
\hline & \multicolumn{2}{|l|}{ VLDL } & \multicolumn{2}{|l|}{ LDL } & \multicolumn{2}{|c|}{ VLDL+LDL } & \multicolumn{2}{|l|}{ HDL } & \multicolumn{2}{|l|}{ Summe } \\
\hline & \multirow[b]{2}{*}{$\begin{array}{l}\text { Mittel- } \\
\text { wert }\end{array}$} & \multicolumn{5}{|c|}{$\mathrm{mg} / \mathrm{dl}$} & \multirow{2}{*}{\multicolumn{2}{|c|}{. }} & \multirow[b]{2}{*}{$\begin{array}{l}\text { Mittel- } \\
\text { wert }\end{array}$} & \multirow[b]{2}{*}{ \pm} \\
\hline & & \pm & $\begin{array}{l}\text { Mittel- } \\
\text { wert }\end{array}$ & \pm & $\begin{array}{l}\text { Mittel- } \\
\text { wert }\end{array}$ & \pm & & & & \\
\hline $\begin{array}{l}\text { Cholesterinester } \\
\text { Triglyceride } \\
\text { Cholesterin } \\
\text { Freie Fettsäuren } \\
\text { Phospholipide } \\
\text { Gesamtcholesterin } \\
\text { Gesamtlipide }\end{array}$ & $\begin{array}{r}21,4 \\
21,6 \\
11,2 \\
5,3 \\
19,8 \\
23,1 \\
79,3\end{array}$ & $\begin{array}{l}1,4 \\
1,9 \\
1,0 \\
0,5 \\
2,0 \\
1,8 \\
5,7\end{array}$ & $\begin{array}{l}231 \\
120 \\
79,5 \\
18,0 \\
145 \\
209 \\
594\end{array}$ & $\begin{array}{r}1,7 \\
6,1 \\
7,4 \\
0,5 \\
13,5 \\
6,5 \\
25,8\end{array}$ & $\begin{array}{l}253 \\
142 \\
90,7 \\
23,2 \\
165 \\
232 \\
673\end{array}$ & $\begin{array}{r}0,3 \\
8,0 \\
8,4 \\
0,1 \\
15,5 \\
8,2 \\
31,5\end{array}$ & $\begin{array}{l}53,3 \\
13,2 \\
13,6 \\
21,4 \\
57,6 \\
43,4 \\
159\end{array}$ & $\begin{array}{r}8,7 \\
0,1 \\
3,6 \\
8,3 \\
17,6 \\
1,3 \\
20,6\end{array}$ & $\begin{array}{r}306 \\
155 \\
104 \\
45 \\
223 \\
276 \\
832\end{array}$ & $\begin{array}{r}9,1 \\
7,9 \\
12,0 \\
8,2 \\
33,1 \\
6,9 \\
52,1\end{array}$ \\
\hline
\end{tabular}

in $\alpha_{2}$-Position wandernden VLDL eine langsamer wandernde $\beta$-VLDL-Bande nachweisbar ist (43). Wie von $K o o k$ et al. (44) gezeigt wurde, werden durch Heparin$\mathrm{MgCl}_{2}$ bevorzugt diese $\beta$-VLDL gefällt. Die relative Lipidzusammensetzung der gefällten VLDL (Tab: 1 ) stimmt gut mit Angaben anderer Autoren über die Lipidzusammensetzung der IDL $(\mathrm{d}=1,006-1,019 \mathrm{~g} / \mathrm{ml})(37,43) \mathrm{bzw}$. der VLDL und IDL beim Typ III (45) überein. Nach neueren Untersuchungen kann der Anteil an IDL, dem physiologischen Abbauprodukt der VLDL $(37,43)$ in VLDL-Präparationen von Normalpersonen bis zu $50 \%$ betragen (45). Von der Lipidzusammensetzung und vom Fällungsmechanismus her ist daher die Annahme berechtigt, daß bei Fällung mit Heparin- $\mathrm{MgCl}_{2}$ bevorzugt kleinere, bereits metabolisierte VLDL-Partikel (,remnant VLDL“ und IDL) erfaßt werden.

Vergleicht man die für die beschriebene Fällungsmethode erhaltenen Variationskoeffizienten (Tab. 2) mit Angaben von Carlson über Variationskoeffizienten der Ultrazentrifugen-Methode (7), so liegen die Werte für LDLGesamtcholesterin, LDL-Triglyceride und HDL-Gesamtcholesterin, HDL-Triglyceride in derselben Größenord nung (5-8\%). Von anderen Autoren wurden für andere Mikromethoden Variationskoeffizienten von $4 \%$ für LDLGesamtcholesterin $(46,47)$ und 2\% (46) bzw. 7\% (47) für HDL-Gesamtcholesterin angegeben.

Bei den in geringer Konzentration vorkommenden VHDL liegen die Variationskoeffizienten zwischen 10-15\% und bei den VLDL zwischen 15-20\%. Bei niedrigem Gesamtlipid der VLDL $(<30 \mathrm{mg} / \mathrm{dl})$ nähert sich die Methode bei der dünnschichtchromatographischen Bestimmung der Lipidverteilung der unteren Nachweisgrenze, wodurch der relative Fehler größer wird. Jedoch auch mit anderen Methoden wurden für das VLDL-Gesamtcholesterin Variationskoeffizienten von $10 \%$ (46), $13 \%$ (47) und 21\% (46) berichtet. Die Absolutwerte für das VLDL-Gesamtcholesterin lagen bei einer MikroAirfuge-Methode (46) 30-40\% unter und bei einer elektrophoretischen Methode kombiniert mit gaschromato= graphischen Cholesterinbestimmungen (47) $50 \%$ über den entsprechenden Werten nach Ultrazentrifugation.
Die Unterschiede in den VHDL - höherer Gehalt an freien Fettsäuren und niedrigerer Gehalt an Phospholipiden und Cholesterinestern bei der Ultrazentrifugenmethode - scheinen methodisch bedingt zu sein. Lipolyse während der sequentiellen Ultrazentrịfugation kann kaum vermieden werden. Der gegenüber anderen Methoden $(17,31,38)$ (Tab. 1) geringfügig höhere Phospholipidgehalt in den VHDL könnte auf eine unvollständige Fällung der HDL zurückzuführen sein. Hierfür spricht auch die Beobachtung, daß der Überstand nach HDL-Fällung in einzelnen Fällen noch leicht trübe war. Zur Umgehung dieser Fehlerquelle wird empfohlen, die Zentrifugationsdauer auf $10 \mathrm{~min}$ zu erhöhen.

Für die Mehrzahl klinischer Fragestellungen erscheint jedoch eine Differenzierung der Lipoproteine in HDL und VHDL nicht erforderlich. Der Uberstand nach LDLFällung kann als HDL-Äquivalent ( $\alpha$-Lipoproteine) angesehen werden. Diese für den klinischen Gebrauch empfohlene zweiśchrittige Fällungsmethode hat im Vergleich mit anderen Methoden zur Lipoproteinanalyse folgende Vorteile:

1. Sie ermöglicht eine approximative Bestimmung der VLDL, LDL und HDL ohne Ultrazentrifugation.

2. Die Lipide dieser Lipoproteine können quantitativ bestimmt werden.

3. Die Variationskoeffizienten sind für die Hauptlipidkomponenten der LDL und HDL mit Variationskoeffizienten nach Ultrazentrifugation vergleichbar.

4. Die Methode ist eine Mikromethode und benötigt nur $50 \mu \mathrm{l}$ Serum.

5. Sie ișt an Geräte adaptiert, die in den meisten Kliniklaboratorien vorhanden sind.

6. Sie ist kostensparend und leicht zu erlernen.

7. Es können auch tiefgefrorene Serumproben analysiert werden.

Die Methode ist geeignet zur Charakterisierung von Hyperlipoproteinämien, vor allem bei leichten Formen, bei denen die Typisierung aufgrund grenzwertiger oder minimaler Triglycerid- und/oder Cholesterinwerte pro- 
blematisch ist. So kann durch quantitative LDL-Cholesterin-Bestimmung und VLDL-Triglycerid-Bestimmung zwischen den häufigsten Hyperlipoproteinämie-Typen IIa, IIb und IV differenziert werden (siehe Beispiel Tab. 3).

\section{Literatur}

1. Kannel, W. B., Castelli, W. P., Gordon, T. \& McNamara, P. M. (i971): Ann. Intern. Med. 74, 1-12.

2. Gustavson, A., Elmfeldt, D., Wilhelmsen, L. \& Tibblin, G. (1972): Circulation 46, 709 $\rightarrow 716$.

3. Goldstein, J. L., Hazzard, W. R., Schrott, H. G., Bierman, E. L., Motulsky, A. G., Levinski, M. J. \& Campell, E. D. (1973): J. Clin. Invest. 52, 1533-1543.

4. Carlson, L. A. \& Ericsson, M. (1975): Atherosclerosis 21, 435-450.

5. Hatch, F. T. \& Lees, R. S. (1968): Adv. Lipid Res. 6, 1-68.

6. Lindgren, F. T., Jensen, L. C. \& Hatch, F. T. (1972): "Blood Lipids and Lipoproteins: Quantitation, Composition and Metabolism", (Nelson, G. J., ed.) Wiley-Interscience, New York, pp. 181-274

7. Carlson, K. (1973): J. Clin. Pathol. 26, Suppl. 5, 32-37.

8. Fredrickson, D. S., Levy, R. I. \& Lees, R. S. (1967): New Engl. J. Med. 276, 34-44, 94-103, 148-156, 215-224, 273-281.

9. Beaumont, J. L., Carlson, L. A., Cooper, G. R., Fejfar, Z. Fredrickson, D. S. \& Strasser, T. (1972): Circulation 45, 501-508.

10. Greten, H., Seidel, D., Walter, B. \& Kolbe, J. (1970): Dtsch. Med. Wochenschr. 95, 1716-1723.

11. Fredrickson, D. S. (1975): Circulation 51, 209-211.

12. Iammarino, R. M. (1975): Clin. Chem. 21, 300-308

13. Lees, R. S. (1970): Science 169, 493-495.

14. Durrington, P. N., Whicher, J. T., Warren, C., Bolton, C. H. \& Hartog, M. (1976): Clin. Chim. Acta 71, 95-108.

15. Cheung, M. C. \& Albers, J. J. (1977): J. Clin. Invest. 60, 43-50.

16. Cornwell, D. G. \& Kruger, F. A. (1961): J. Lipid Res. 2, $110-134$.

17. Burstein, M., Scholnick, H. R. \& Morfin, R. (1970): J. Lipid Res. 11, 583-595.

18. Miller, G. J. and Miller, N. E. (1975): Lancet $I, 16-19$.

19. Rhoads, G. G., Gulbrandsen, C. L. \& Kagan, A. (1976): New Engl. J. Med. 294, 293-298.

20. Miller, G. J., Miller, N. E. \& Ashcroft, M. T. (1976): Clin. Sci. Mol. Med. 51, 475-482.

21. Gordon, T., Cástelli, W. P., Hjortland, M. C. Kannel, W. B. \& Dawber, T. R. (1977): Amer. J. Med. 62, 707-714.

22. Castelli, W. P., Doyle, J. T., Gordon, T., Hames, C. G., Hjortland, M. C., Hulley, S. B. Kagan, A. \& Zukel, W. J. (1977): Circulation 55, 767-772.

23. Rössner, S., Kjellin, K. G., Mettinger, K. L., Siden, A. and Söderström, C. E. (1978): Lancet $I, 577-579$.

24. Glueck, C. J. (1976): Artery 2, 196-198.

25. Burstein, M. \& Samaille, J. (1960): Clin. Chim. Acta 5, 609.
Weiter vereinfacht auf eine einstufige $\beta$-Lipoproteinfällung kann die Methode auch zur Bestimmung des Cholesterins der HDL bei Risikogruppen und wegen der geringen benötigten Serummenge auch bei Neugeborenen angewandt werden.
26. Bachorik, P. S., Woos, P. D., Albers, J. J., Steiner, P., Dempsey, M., Kuba, K., Warnick, R. \& Karlsson, L. (1976): Clin. Chem. 22, 1828-1834.

27. Ishikawa, T. T., Brazier, J. B., Steiner, P. M., Stewart, L. E., Gartside, P. S. \& Glueck, C. J. (1976): Lipids 11, 628-633

28. Wilson, D. E. \& Spiger, M. (1973): J. Lab. Clin. Med. 82, $473-482$.

29. Ononogbu, I. C. \& Lewis, B. (1976): Clin. Chim. Acta 71, 397-402.

30. van Gent, C. M. (1968): Z. Anal. Chem. 236, 344-350.

31. Egge, H., Murawski, U., Müller, J. \& Zilliken, F. (1970): Z. Klin. Chem. Klin. Biochem. 8, 488-491.

32. Havel, R. J., Eder, H. A. \& Bragdon, J. H. (1955): J. Clin. Invest. 34, 1345-1353.

33. Folch, J., Lees, M. \& Sloane Stanley, G. H. (1957): J. Biol. Chem. 226, 497-509.

34. Zlatkis, A., Zak, B. \& Boyle, A. J. (1953): J. Lab. Clin. Med. 41, 486-492.

35. Lutmer, R. F., Parson, D., Glueck, C. J., Morrison, J. A., Stewart, L., Brazier, J. B., Buncher, C. R. \& Ishikawa, T. T. (1974): J. Lipid Res. 15, 611-614.

36. Kostner, G. M. (1976): Clin. Chem. 22, 695.

37. Augustin, J., Middelhoff, G. \& Brown, W. V. (1976): „Fettstoff wechsel", (Schettler, G., Greten, H., Schlierf, G. \& Seidel, D. eds.) Handbuch der Inneren Medizin (Schwiegk, H. ed.), Springer-Verlag, Berlin-Heidelberg-New York, pp. 219-264.

38. Skipski, V. P. (1972): „Blood Lipids and Lipoproteins: Quantitation, Composition and Metabolism", (Nelson, G. J. ed.) Wiley-Interscience, New York, pp. 471-583.

39. Cham, B. E. (1976): Clin. Chem. 22, 1812-1816.

40. Kim, Y. C. \& Nishida, T. (1977): J. Biol. Chem. 252, 12431249.

41. Shelburne, F. A. \& Quarfordt, S. H. (1977): J. Clin. Invest. $60,944-950$.

42. Mahley, R. W. \& Innerarity, T. (1977): J. Biol. Chem. 252, 3980-3986.

43. Pagnan, A., Havel, R. J., Kane, J. P. \& Kotite, L. (1977): J. Lipid Res. 18, 613-622.

44. Kook, A. I., Eckhaus, A. S. \& Rubinstein, D. (1970): Can. J. Biochem. 48, 712-724.

45. Gianturco, S. H., Gotto, A. M. jr., Jackson, R. L., Patsch, J. R., Sybers, H. D., Taunton, O. D., Yeshurun, D. L. \& Smith, L. C. (1978): J. Clin. Invest. 61, 320-328.

46. Bronzert, T. J. \& Brewer, H. B. jr. (1977): Clin. Chem. 23, 2089-2098.

47. Heuck, C. C., Nothelfer, A., Raetzer, H. \& Schlierf, G. (1977): J. Lipid Res. 18, 259-263.
Prof. Dr. H. Egge

Institut für Physiologische Chemie der Universität Bonn

Nußallee 11

5300 Bonn 


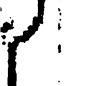

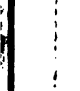

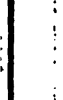

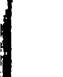

:

( $\cdots$

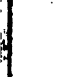

